\title{
Biodiversity for our future
}

\author{
Yukio Yokota $\cdot$ Valeria Matranga
}

Published online: 6 March 2010

(C) Springer Science+Business Media, LLC 2010

\begin{abstract}
This editorial introduces a Special Issue on the topic "Alternative Models for Marine Toxicological Investigations". Today, the terrestrial and marine environments are being changed by anthropogenic activities at a very high rate. Rapid change, coupled with uncontrolled exploitation of natural resources e.g., lands, mines, waters, organisms etc., as well as industrialization, has resulted in a grave situation for the global environment. On the Year of Biodiversity this special issue summarises the current situation in the marine environment and discusses innovative ecotoxicological tests related to the protection of marine organisms.
\end{abstract}

\section{Introduction}

Due to pollution and changes in the global environment, a variety of organisms are currently endangered, productivity of biota is decreasing and some species are becoming extinct. Our human society is based on the productivity of biota and the conservation of biodiversity. The latter can be measured as genetic diversity, species diversity and ecosystem diversity, all of which are crucial to make our society sustainable. Our own survival is therefore dependent on responsible sustainable use of the environment.

\section{Y. Yokota}

Department of Information Science and Technology, Aichi Prefectural University, Nagakute, Aichi 480-1198, Japan e-mail: yokota@ist.aichi-pu.ac.jp

\section{Matranga ( $\square)$}

Consiglio Nazionale delle Ricerche, Istituto di Biomedicina e Immunologia Molecolare "Alberto Monroy", Via Ugo La Malfa 153, 90146 Palermo, Italy

e-mail: matranga@ibim.cnr.it
Protection of the marine environment is partly achieved by evaluating the hazard of pollutants to marine organisms, and the discipline of ecotoxicology has been central to providing such data, as well as the scientific tools for monitoring pollution. Marine ecotoxicology is applying new techniques including ecotoxicogenomics, ecotoxicoproteomics, biochemical ecology, and others (for a review on the argument see Snape et al. 2004).

These scientific techniques and their application to ecotoxicology, as well as other new fields of scientific investigation, are expanding our perspectives on environmental protection from macroscopic to microscopic phases and vice versa. In addition, these new techniques are expected to aid hazard assessment, and will therefore enable the precautions needed to protect the environment against a variety of agents (e.g., mutagens, Chen and White 2004). These tools may also define biomarkers of toxicity to humans (Galloway 2006; Hagger et al. 2008). For example, 'omics technology could precisely identify the patterns of expression of characteristic genes and proteins in organisms present in a polluted environment. The identification and establishment of marker genes specifically expressed in organisms exposed to environmental pollutants, may be of great importance in predicting changes in biodiversity.

Furthermore, there is a general need for new emerging scientific disciplines and technologies to foretell the future of the marine environment, the marine organisms contained therein, the future impacts that may occur. In this regard, the use of numerical analyses such as probababilistic risk assessment, multi-variate statistics, linear and non-linear simulations; all aided with new computation methods (e.g., systems toxicology), will significantly contribute to the assessment of chemical substances in the environment. Important areas of application are: (a) the quantitative 
evaluation of animal behavior, to predict and/or forecast long-term environmental changes; (b) management of the natural environment by continuously monitoring its transition; and, (c) restoration of the damaged environment. Here, it must be remembered that the counter measures to environmental perturbation caused by anthropogenic activities might generate unexpected and unintended impacts on organisms as well.

\section{Scope of the special issue}

The genesis of this Special Issue was the 3rd Bilateral Seminar Italy-Japan "Physical and Chemical Impacts on Marine Organisms-Seeking Sustainability and Postgenomics" that was held 24-27th November, 2008, in Mielparque-Nagoya, Nagoya, Japan. The purpose of the 3rd Bilateral Seminar was to consider model organisms and recent methodologies able to predict the effects of environmental stress factors on marine organisms. The 1st Bilateral Seminar on marine environmental issues took place in Shima, Japan from the 16th to the 19th of November 2004 (Marine Biology, vol. 149, No. 1, 2006) and continued with the 2nd Bilateral Seminar held in Palermo, Italy from the 22nd to 25th of November 2006 (Cell Biology and Toxicology, vol. 24, No. 6, 2008). A 4th Bilateral Seminar is currently in the planning stage and will emphasize new approaches to investigate water temperature and acidification in relation to marine organism biodiversity.

\section{Summary of papers appearing in this special issue}

After an independent peer-reviewing process, twelve papers from oral presentations at the 3rd Bilateral Seminar were accepted for publication in this Special Issue. In addition, an invited paper was added in order to cover an aspect of physical impacts on marine ecosystems that, in our minds, is a very important topic. The ongoing decrease in the $\mathrm{pH}$ of the Earth's oceans, also referred to as ocean acidification (OA), caused by the uptake of anthropogenic carbon dioxide from the atmosphere has become a major concern for marine ecosystems (Guinotte and Fabry 2008). $\mathrm{OA}$ is expected to have deleterious impacts on many organisms living in the sea, especially those with calcifying structures including corals (Hoegh-Guldberg et al. 2007), echinoderms (Todgham and Hofmann 2009) and others. Apart from this, OA is suspected to have dangerous effects on marine animal biodiversity (Jackson 2008). A brief description of each paper follows.

The invited review paper by Dupont et al., entitled "Impact of near-future ocean acidification on echinoderms" summarizes what is known about the impact of OA on this endangered phylum. Using meta-analysis, the authors approach the identification of bottlenecks in physiological processes, critical life-cycle stages, and gaps in our general knowledge.

"Dioxins and organohalogen contaminants in the AsiaPacific region" by Tanabe and Minh comprehensively reviews investigations by the authors over the past three decades on the distribution, sources, temporal trends and toxic impacts of organohalogen pollutants in Asia-Pacific region.

"Impaired megabenthic community structure caused by Summer hypoxia in a eutrophic coastal bay" by Kodama et al., notes that hypoxia significantly impairs the megabenthic community of the Tokyo bay, a eutrophic coastal area rich in the megabenthic community, located in a highly urbanized and industrialized area. This investigation contributes to understanding of the marine environment and the organisms in littoral zones and coastal bay areas (which are of global concern), as well as the need to establish counter measures against environmental deterioration.

"Toxigenic effects of diatoms on grazers, phytoplankton and other microbes: A review", by Ianora and Miralto, reviews the literature on the possibility that diatoms produce cytotoxic compounds responsible of reduced cell growth and teratogenenesis of their predators, mostly copepods. Such toxins, collectively termed oxylipins and including the polyunsaturated aldehydes (PUAs), also act as allelopathic agents against other phytoplankton cells, signalling population-level cell death and termination of blooms. Therefore, by virtually affecting the growth of competitors, diatoms might have impacting consequences on food web structure and community composition. Interestingly, the chemical complexity of diatoms, never suspected more than 10 years ago, may have been driven by the necessity to avoid co-evolution by predators to detoxify oxylipins.

"Swimming speed alteration of cysts-forming marine organisms, Artemia salina and Brachionus plicatilis, as a sub-lethal behavioural end-point for ecotoxicological surveys" by Garaventa et al., standardized, using two model organisms, a Swimming Speed Alteration test (SSA test), which can detect stress at sub-lethal toxic substances concentrations. The use of such an easy and quick test is proposed for ecotoxicological studies and environmental monitoring programs.

"Dose-dependent effects of chlorpyriphos, an organophosphate pesticide, on metamorphosis of the sea urchin, Paracentrotus lividus" by Aluigi and coauthors extends the sea urchin embryo model for testing the toxicity of compounds (insecticides) to metamorphosis-competent larvae. The model is very sensitive not only for testing neurotoxic 
xenobiotics, but also for monitoring the effects of endocrine disrupters (EDs) dispersed in the sea, given the fact that larval tissues are directly exposed to the environment. Endangering sea urchin reproductive success caused by the spreading of pesticides from the agricultural sites located near the shallow-water coasts during the early springtime, when echinoids are fertile, is discussed.

"Embryonic development and gene expression affected by X-rays in the model system Paracentrotus lividus" by Matranga et al., describes how sea urchin embryos can be used to assess the risk of exposure to ionizing radiation. The problem of nuclear contamination assumes an important significance today, not only because of the fortunately rare occurrence of accidents; but, most importantly, because of the nuclear waste excess and improper stocking. The findings indicate the sea urchin embryo model system as a sensible biosensor of $\mathrm{X}$-radiations and propose its use as an alternative model for investigations of the underlying mechanisms of action.

"Chemical fate and biological effects of several Endocrine Disrupters Compounds in two echinoderm species" by Sugni et al., reviews the most significant results of ecotoxicological tests developed by the authors to assess the effects of EDs on two echinoderm species: the echinoid Paracentrotus lividus and the crinoid Antedon mediterranea. New and previous results are integrated to give an original view on: chemical fate in the experimental aquaria, hormonal responses to the chemicals and effects on reproductive and regenerative parameters. In addition, the biological effects measured were compared to a predictive mathematical model, allowing the evaluation of the daily dose of the chemical entering in each test animal and offering a realistic overview of the actual chemical intake during the test. The multidisciplinary method is proposed for the evaluation and comprehension of the global effects of EDs and is predicted to allow more realistic estimates on the environmental conditions.

"Sea urchin embryos as an in vivo model for the assessment of manganese toxicity: developmental and stress response effects" by Pinsino et al., demonstrated that, despite its essential role in physiological cell functions, manganese $(\mathrm{Mn})$ might have a negative impact on the sea urchin embryogenesis, depending on its concentrations in the environment. At experimental concentrations from 2 to 4 orders of magnitude higher that those found in nature development to the pluteus stage was greatly impaired. Nevertheless, embryos showed an elevated tolerance to $\mathrm{Mn}$, as at low concentrations they were able to accumulate it into cells. The finding that no apoptosis was induced by $\mathrm{Mn}$ exposure, confirmed the notion that stress proteins (hsp70 and hsp60) play a role in protecting embryos against apoptosis. The authors indicate hsps are sensitive markers for predicting environmental stress and confirm the sea urchin embryo as a good model for ecotoxicological studies.

"Distinct embryotoxic effects of lithium appeared in a new assessment model of the sea urchin: the whole embryo assay and the blastomere culture assay" by Kiyomoto et al., shows that the effect of chemicals can vary dependent on the experimental designs and conditions. The effect of $\mathrm{LiCl}$ on the whole sea urchin embryo is different from that on the dissociated blastomeres, suggesting the importance of the design of experimental conditions for ecotoxicological analysis and understanding of the experimental results.

"Establishment of a polyclonal antibody against the retinoid X receptor of the rock shell Thais clavigera and its application to rock shell tissues for imposex research" by Horiguchi, et. al., deals with establishment and characterization of a polyclonal antibody against Thais clavigera retinoid $X$ receptor which is suggested to be involved in the induction of male-type genitalia by organotin. The antibody is expected to be an effective tool for the analysis of imposex induction in gastropods.

"Isolation and characterization of a cDNA encoding a heat shock protein 70 from a sterile mutant of Ulva pertusa (Ulvales, Chlorophyta)", by Tominaga et al., describes the isolation and characterization of a cDNA clone encoding heat shock protein 70 (hsp70) from a sterile strain of $U$. pertusa. Expression of hsp70 increases rapidly in the condition of high temperature and gradually in the presence of heavy metals, including cadmium, copper and lead. This sterile strain of $U$. pertusa is a useful model organism for the investigation of the stress response in algae, considering the ease of maintenance in the laboratory and the availability of genomic and proteomic information.

"Introduction of foreign DNA into the water flea, Daphnia magna, by electroporation", by Kato et al., is the first report on the introduction of foreign DNA into the water flea, D. magna. This organism is an important standard test organism for ecotoxicological investigations. This method, together with the emerging genome sequences, will be useful for expanding our use of daphnids as molecular tools in ecotoxicology.

\section{Concluding remarks}

Papers contained in this issue reported sensitive and innovative methods used to obtain information on the effects of selected pollutants present in the marine environment, or dispensed in laboratory trials. Stressors, included also physical changes of the water milieu, such as acidic pH (Dupont et al., this issue), hypoxia (Kodama et al., this issue) and X-rays (Matranga et al., this issue). Chemicals comprised: oxilipins (Ianora and Miralto, this issue), antifouling agents/pesticides (Garaventa et al., this 
issue; Aluigi et al., this issue), EDs (Sugni et al., this issue; Horiguchi et al., this issue) and metals (Pinsino et al., this issue; Kiyomoto et al, this issue; Tominaga et al, this issue). In one case a genetic manipulation approach has been developed to be used in ecotoxicogenomics studies (Watanabe et al. 2008; Kato et al., this issue). The "fil rouge" of this issue is that the ecotoxicological tests described herewith are innovative, as they propose model organisms which have not been classically used in the context of risk assessment, or are rapid methods as measurements are made on relatively fast developmental/physiological processes, or can be assessed using quick molecular biomarkers tests, or a combination of the two. In addition, integrated approaches and computation methods will ensure a better understanding of spatial/temporal resolution of exposure and/or effects (Sugni et al., this issue). Each test is designed to rapidly screen many pollutants in batteries; some are expected to be patented soon offering good/easy methods to local agencies controlling environmental health. We believe that all the alternative models described in this issue will aid to the understanding of toxic effects of new emerging pollutants, and, in this respect we predict model organisms having their genome available, will expand our knowledge and contribute to government(s) policy makers adopting decisions for the survival and sustainable future of the marine environment.

Acknowledgments The organizers of the seminar expresses their gratitude to UNESCO Man and Biosphere Programme for the high patronage, the Consiglio Nazionale delle Ricerche, Japan Society for Promotion of Science, Istituto Superiore per la Protezione e la Ricerca
Ambientale, Dipartimento di Scienze Ambientali-Università di Siena and Daiko Foundation for their financial support, and to all participants in the Bilateral Seminar for a successful program.

\section{References}

Chen G, White PA (2004) The mutagenic hazards of aquatic sediments: a review. Mutat Res 567:151-225

Galloway TS (2006) Biomarkers in environmental and human health risk assessment. Mar Pollut Bull 53:606-613

Guinotte JM, Fabry VJ (2008) Ocean acidification and its potential effects on marine ecosystems. Ann NY Acad Sci 1134:320-342

Hagger JA, Jones MB, Lowe D, Leonard DR, Owen R, Galloway TS (2008) Application of biomarkers for improving risk assessments of chemicals under the Water Framework Directive: a case study. Mar Pollut Bull 56:1111-1118

Hoegh-Guldberg O, Mumby PJ, Hooten AJ, Steneck RS, Greenfield P, Gomez E, Harvell CD, Sale PF, Edwards AJ, Caldeira K, Knowlton N, Eakin CM, Iglesias-Prieto R, Muthiga N, Bradbury RH, Dubi A, Hatziolos ME (2007) Coral reefs under rapid climate change and ocean acidification. Science 318:1737-1742

Jackson JB (2008) Colloquium paper: ecological extinction and evolution in the brave new ocean. Proc Natl Acad Sci USA 105:11458-11465

Snape JR, Maund SJ, Pickford DB, Hutchinson TH (2004) Ecotoxicogenomics: the challenge of integrating genomics into aquatic and terrestrial ecotoxicology. Aquat Toxicol 67:143-154

Todgham AE, Hofmann GE (2009) Transcriptomic response of sea urchin larvae Strongylocentrotus purpuratus to $\mathrm{CO}_{2}$-driven seawater acidification. J Exp Biol 212:2579-2594

Watanabe H, Kobayashi K, Kato Y, Oda S, Abe R, Tatarazako N, Iguchi T (2008) Transcriptome profiling in crustaceans as a tool for ecotoxicogenomics: Daphnia magna DNA microarray. Cell Biol Toxicol 24:641-647 